\title{
Characterization of nanograined powder samples using the Rietveld method applied to electron diffraction ring patterns
}

\author{
A. Serafini, ${ }^{1, a)}$ L. Lutterotti, ${ }^{2}$ S. Gross, ${ }^{3}$ and S. Gialanella ${ }^{2}$ \\ ${ }^{I}$ Dipartimento di Chimica, Materiali e Ingegneria Chimica "Giulio Natta", Politecnico di Milano, Italy \\ ${ }^{2}$ Dipartimento di Ingegneria Industriale, Università degli Studi di Trento, Italy \\ ${ }^{3}$ Dipartimento di Scienze Chimiche, Istituto di Chimica della Materia Condensata e di Tecnologie per l'Energia - ICMATE-CNR, \\ Università degli Studi di Padova, Italy
}

(Received 15 September 2016; accepted 6 March 2017)

\begin{abstract}
A full-pattern fitting procedure based on the Rietveld method was applied to electron diffraction ring patterns of a two-phase system, exhibiting the co-presence of zinc sulfide (sphalerite) and zinc oxide (Wurtzite). Bright and dark field (DF) images reveal the presence of micrometric aggregates, composed of quasi-spherical nanosized crystallites. These conventional transmission electron microscopy imaging methods provide a general morphological characterization of the specimens although, in the present case, they are not suitable for a detailed characterization of the microstructural features of the analyzed samples. Owing to the overlap and broadening of the diffraction rings of the two phases, DF images cannot provide a satisfactory picture of the individual crystallites of each single phase. To overcome this limit, the mentioned Rietveld approach was applied to model the electron diffraction data. The crystalline domain size and relevant shapes for both phases were successfully evaluated using the proposed methodological approach. The excellent results obtained in the microstructural characterization of the nanostructured multiphase samples demonstrate the capability of this technique, that may represents a fully quantitative method for the routine characterization of crystalline nanomaterials. (C) 2017 International Centre for Diffraction Data.
\end{abstract}

[doi:10.1017/S0885715617000343]

Key words: electron diffraction, Rietveld method, TEM, nanograins, quantitative analysis, size-strains

\section{INTRODUCTION}

Nowadays nanomaterials are widely diffused and they are increasingly studied for being applied in many different fields, such as health and medicine (Jain et al., 2008), food products (Silvestre et al., 2011), textiles (Yetisen et al., 2016), electronics (Simon and Gogotsi, 2008), energy, and environment (Klaine et al., 2008). For regulatory purposes, the European Commission has recently issued a common definition of the term "nanomaterial" (Linsinger et al., 2012): "nanomaterial means a natural, incidental or manufactured material containing particles, in an unbound state or as an aggregate or as an agglomerate and where, for $50 \%$ or more of the particles in the number size distribution, one or more external dimensions is in the size range 1-100 $\mathrm{nm}$ ". In the proposed legislation, the particle size distribution is present as a fundamental parameter for deciding whether a certain material can be classified as a nanomaterial or not. Electron microscopy, scanning probe microscopy, X-ray diffraction (XRD), and dynamic light scattering are some of the measurement methods available for the determination of the characteristic dimensions of nanomaterials (Hassellöv et al., 2008; Baer et al., 2010; Linkov et al., 2013). Certainly, transmission electron microscopy (TEM) is one of the best tools for the characterization of nanostructured systems. It is routinely used for the investigations on grain size and other microstructural aspects down to sub-nanometric scale (Lascialfari et al., 2014; Giorgetti et al., 2015). On

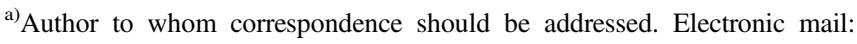
andrea.serafini@polimi.it
}

some occasions, diffraction contrast imaging, based on bright field (BF) and dark field (DF) micrographs, does not allow to determine unambiguously the microstructure, e.g., particle dimension and shape, defect structures, etc. of nanosized materials. In particular, these methods may fail in the presence of agglomerated aggregates of nanoparticles (Dieckmann et al., 2009; Bell et al., 2012), and multiphase nanocrystalline systems. An alternative approach is proposed herewith, and relies on the attainment of microstructural information directly from the electron diffraction patterns, following a procedure adopted with other diffraction methods, like XRD and neutron diffraction, using a full-pattern fitting approach (Gemmi et al., 2011; Boullay et al., 2014), based on the Rietveld method (Rietveld, 1969). In the present study, a mixture of two phases, namely zinc sulfide $(\mathrm{ZnS})$ and zinc oxide $(\mathrm{ZnO})$ was firstly investigated by means of XRD and conventional TEM diffraction contrast mode: BF/DF TEM and selected area electron diffraction (SAED). Subsequently, a microstructural characterization of the powders was carried out by applying the Rietveld method to the selected area electron diffraction patterns. Finally, the results obtained with the two methodologies have been comparatively discussed, even as concerns the phase quantification on different length scales.

\section{MATERIALS AND METHODS}

\section{A. TEM characterization}

TEM analysis was carried out using a Philips CM12 transmission electron microscope operating at $120 \mathrm{kV}$ and 
equipped with an energy-dispersive X-ray spectroscopy (EDXS) system, occasionally used for localized chemical analyses. The powder samples were ultrasonically suspended in ethanol and a drop of this suspension was deposited onto a 200 mesh holey carbon-coated copper grid. SAED patterns and TEM micrographs, both DF and BF images, were acquired. For the identification of the phases and indexing of the relevant peaks, Process Diffraction software was used (Lábár, 2008, 2009; Lábár et al., 2012). Image analysis of TEM DF micrographs were performed using the ImageJ software (Schneider et al., 2012), quite useful to estimate dimension and shape of coherent scattering domains and relevant statistical distributions. In particular, the mean values and widths of the statistical asymmetric distribution $\left(\sigma_{-}, \sigma_{+}\right)$of crystallites were evaluated by fitting the experimental values with a log-normal function.

\section{B. Rietveld method applied to electron diffraction ring patterns}

The Rietveld analysis of SAED patterns was carried out using the MAUD program (Lutterotti, 2010) using electron atomic scattering factors from Peng et al. (1996). Intensity integration along the Debye rings of the nanopowder SAED pattern was performed using ImageJ plug-in implemented directly in MAUD, which allows us to import twodimensional (2D) diffraction data coming from the TEM camera or imaging-plate detectors directly, preserving the original image coordinate position for each data point (Ischia et al., 2005; Lutterotti et al., 2007, 2014). Using an adequate number of diffraction rings it was possible to correct for possible tilting errors and center displacement directly in the Rietveld refinement (Lutterotti et al., 2014). Two kinds of data-set plotting were used. (1) The 2D plot depicts the azimuthal variation of the diffraction intensity plotted vs. the momentum transfer. (2) Intensity profile obtained summing up all the azimuthal plots (corresponding to a full integration along DebyeScherrer rings). SAED patterns were acquired with the beam stopper inserted to reduce the intensity of the transmitted beam. This masked portion of the image was removed and not considered in data modeling. The background of the diffraction pattern was fitted with a polynomial function and an additional Gaussian peak at $0 \mathrm{Q}$, in order to model the strong low angle signal rise, mainly because of the transmitted beam. In general, no important contribution was detected from the supporting holey carbon film, so that no further background correction was needed (Kim et al., 2009). For the microstructural characterization of crystalline domain size and shape, the instrumental broadening function was determined following the method proposed by Boullay et al. (2014). For this purpose, our reference material was a nanocrystalline $\mathrm{TiO}_{2}$ single-phase powder. The first step of the calibration procedure is to determine the average size and shape of the crystallites using an X-ray powder diffractometer with a known instrumental function. The obtained parameters were used as an input in the analysis of the electron diffraction patterns of the reference material to extract the instrumental peak shape function.

\section{Powder XRD measurements}

The XRD data were collected with a Bruker D8 Advance diffractometer equipped with a Göbel mirror and $\mathrm{CuK \alpha}$ radiation.

For all details on the sample preparation procedure and additional characterization results, the reader is referred to the paper of Dolcet et al. (2015).

\section{RESULTS AND DISCUSSION}

Firstly, the selected nanograined powders, synthesized following the procedure described by Dolcet et al. (2015) were fully characterized by XRD and TEM methods. The analyses were conducted to characterize the microstructure (crystalline domain shape and dimension) of the powders. The indexing of the SAED pattern (Figure 1) reveals the presence of a two phase crystalline system, composed of $\mathrm{ZnS}$, cubic, sphalerite and $\mathrm{ZnO}$, hexagonal, Wurtzite. The SAED pattern exhibits a substantially continuous distribution of the diffracted intensity along the rings. This indicates a reduced crystallite dimension of both phases and the lack of any preferential orientation. Indeed, the DF micrograph shows the presence of nanosized coherently scattering crystalline domains. The displayed crystallites are organized in pseudospherical aggregates, clearly visible in TEM BF micrograph (Figure 1), with dimensions from 50 to $100 \mathrm{~nm}$. On the other hand, the XRD analysis (Figure 2) reveals the presence of one phase only: $\mathrm{ZnS}$ (cubic, sphalerite). The discrepancy between SAED and XRD data can be explained by admitting the formation of a thin $\mathrm{ZnO}$ surface layer on the $\mathrm{ZnS}$ grains with an overall volume fraction below the XRD detection limit. However, $\mathrm{ZnO}$ would significantly contribute to electron diffraction, since this is coming from thinner electron-transparent regions where the oxide layer is a
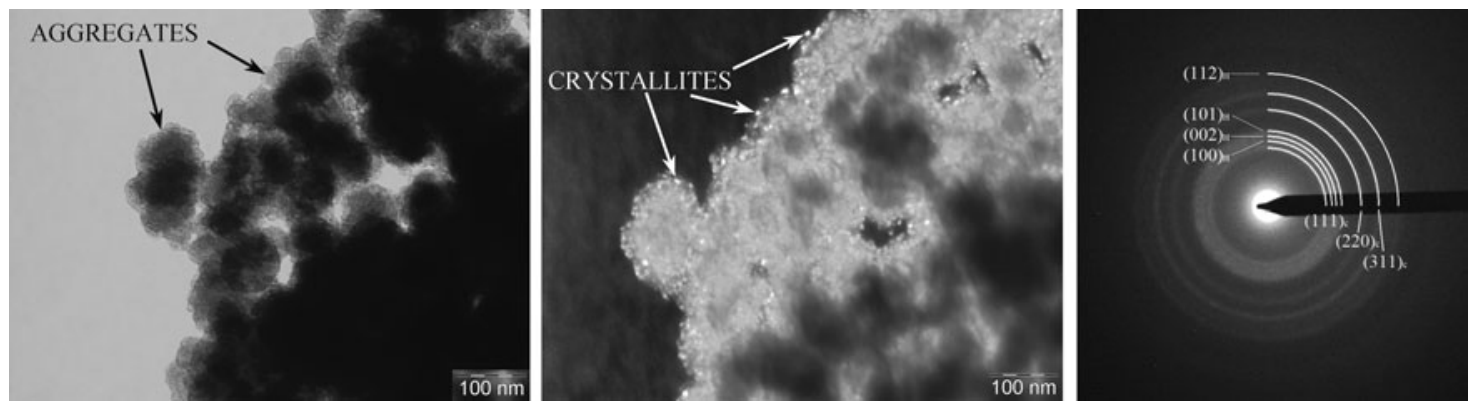

Figure 1. BF TEM micrograph of nanopowder aggregates (left); DF TEM micrograph of the nanopowder aggregates (center); SAED ring pattern (right) of the nanopowder aggregates indexed by Process Diffraction software, showing the presence of two phases: $\mathrm{ZnS}$ and $\mathrm{ZnO}$. 


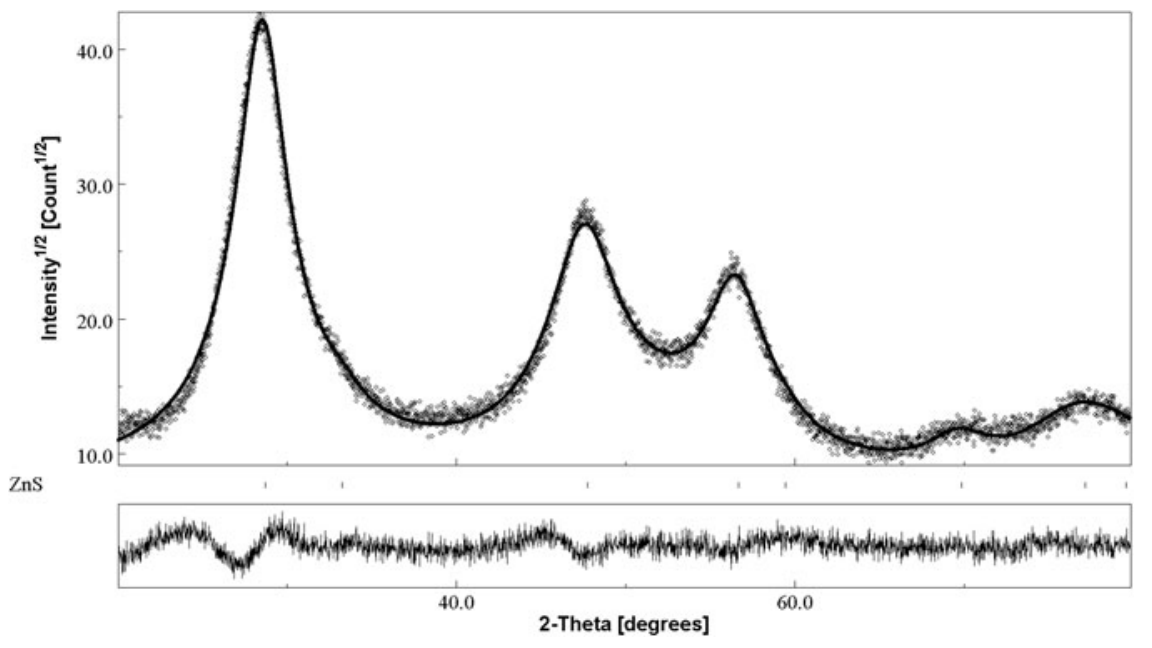

Figure 2. Rietveld method applied to the powder XRD pattern of the nanostructured powders: $\mathrm{ZnS}$ is the only detected phase present. Experimental data points in dotted line; continuous black line: calculated profile. comparatively more important component. The scheme in Figure 3 is depicting this situation concerning the different region sampled in XRD and electron diffraction. In SAED, the size of the analyzed region is determined by the selected area aperture employed for the acquisition. In our case, the electron diffraction pattern of the $\mathrm{ZnS} / \mathrm{ZnO}$ sample was acquired centering the selected area aperture in correspondence of the region visualized in the $\mathrm{BF}$ image in Figure 1, corresponding to a sampled area of about $0.5 \mu \mathrm{m}^{2}$. Moreover, not all of the displayed area would contribute to coherent scattering event but only the thinner, electron transparent regions, with a thickness below $50 \mathrm{~nm}$ approximately. These data indicate that the volume under investigation is well below the fraction of $\mu \mathrm{m}^{3}$ in SAED. On the other hand, common values for the analyzed volume region in XRD are of several $\mathrm{mm}^{3}$. This difference in sampled volumes and the localization of the $\mathrm{ZnO}$ phase on the surface regions explain the discrepancy between the results obtained with the two techniques. From these considerations it turns out how important it is to combine different diffraction methods, such as XRD and SAED, to perform a complete characterization at different length scales of nanostructured materials. Indeed, XRD supplies information on bulk phase content, while SAED is able to provide localized phase identification also at the nanoscale level. Actually, electron diffraction may

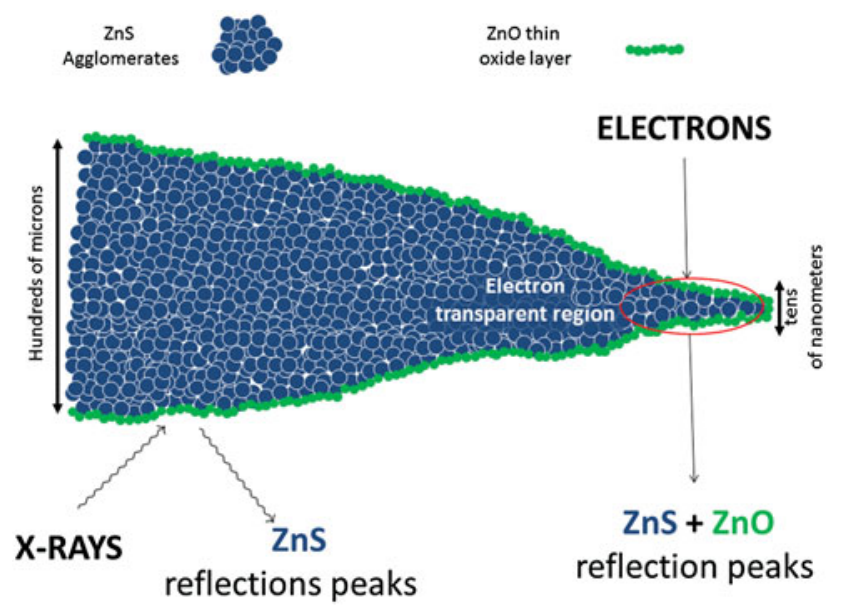

Figure 3. (Color online) Graphical representation of the comparison between $\mathrm{XRD}$ and SAED results. provide quantitative information on nanostructured systems. Recently, Boullay et al. (2014) have shown the potential of the Rietveld method applied to the characterization of nanostructured crystalline samples using SAED data. In this work, we apply for the first time this method to a two-phase sample to achieve significant information on the crystalline domain size and shape of both phases, particularly when phase contrast, i.e., BF and DF imaging, cannot be profitably employed. For a reliable microstructural characterization using Rietveld analysis of SAED patterns, it is recommended to use diffraction patterns with a homogeneous intensity distribution all along the Debye-Scherrer rings, in order to avoid the graininess problem. A homogeneous intensity is obtained when the number of randomly oriented crystallites is large enough. Owing to the reduced analyzable region in SAED this requirement is fulfilled with materials with nanometric crystal grains. The $\mathrm{ZnS} / \mathrm{ZnO}$ system considered herewith represents an appropriate test sample for the proposed approach, since both phases in SAED analysis exhibits homogenous diffraction rings without any graininess (Figure 1). Actually, in this system $\mathrm{ZnO}$ is the undesired passivation layer formed on the surface of the wanted $\mathrm{ZnS}$. Nevertheless, the knowledge of the exact chemical nature and composition of the surface is essential for the development of effective functionalization strategies and for understanding the functional behavior (i.e., catalytic or sensors properties) of this material. The first step of Rietveld analysis was importing SAED images in MAUD and unrolling each one in several intensity profile patterns. Then the crystal structures of the $\mathrm{ZnO}$ and $\mathrm{ZnS}$ phases, previously identified by indexing the relevant SAED (Figure 1), were loaded into the software from COD (Crystallography Open Database) using the Crystallographic Information File (CIF) format (Gražulis et al., 2009). The first refinement cycle was used to adjust the background, the overall scale factor, the instrumental parameters (tilting error and center displacement) and the crystal structure, respectively. In the final step, the microstructure of both phases was refined using the Popa model (Popa, 1998), to take into account the possible shape anisotropy of the analyzed crystalline-scattering domains.

The Rietveld analysis converged quickly to the results reported in Table I. All patterns were treated using kinematical approximation with Blackman two-wave dynamic correction (Blackman, 1939) implemented in MAUD (Boullay et al., 
TABLE I. Results of electron and XRD Rietveld analysis (Figures 2 and 4). The numbers in parentheses denotes the estimated standard deviation in the last digit.

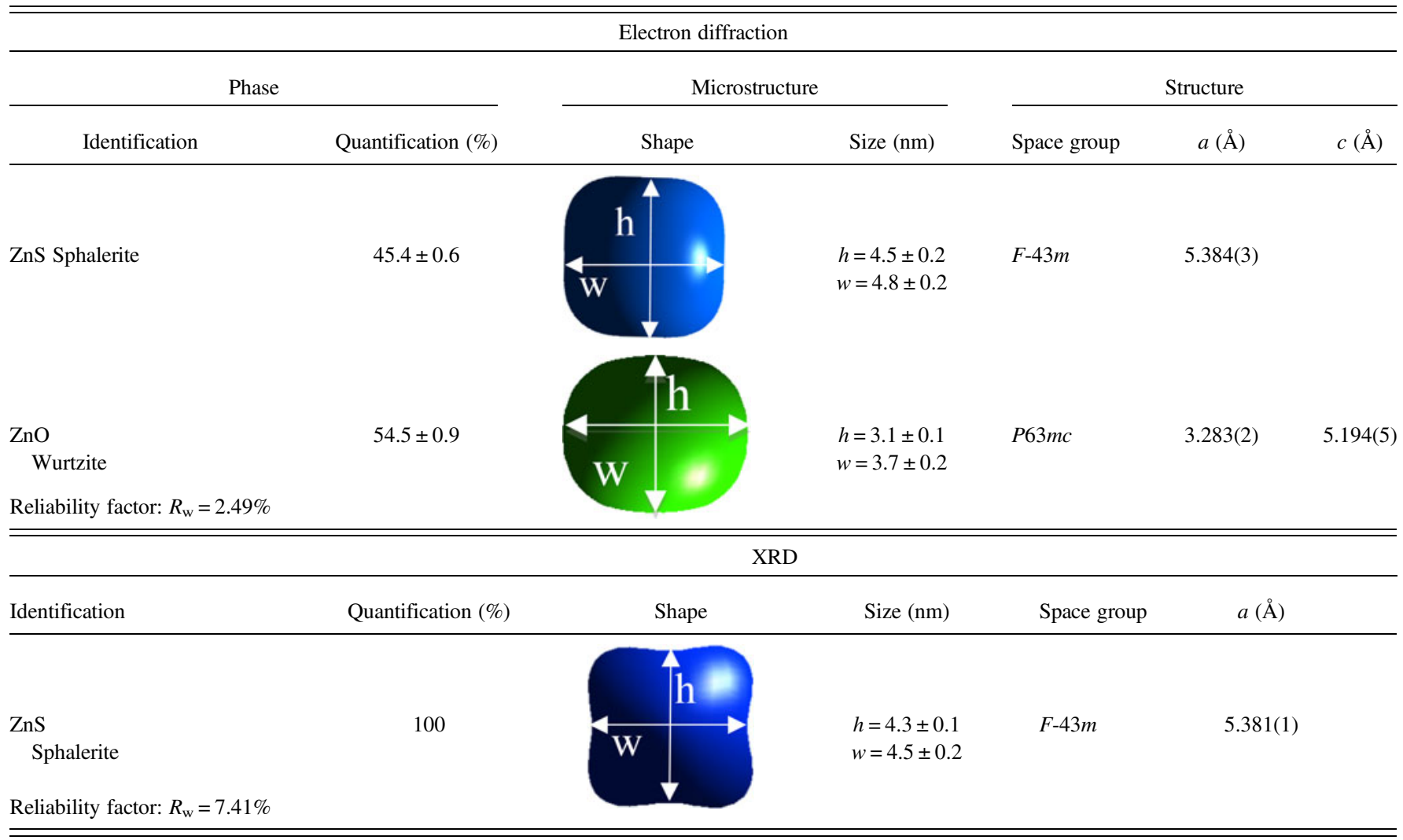

2014). Calculated and experimental diffraction profiles plotted in one and two dimensions are shown in Figure 4 together with the residual curve obtained after the final refinement. It is worth noting that the intensity profile fitting was made by a model that does not take account texture features, since the $\mathrm{ZnS} / \mathrm{ZnO}$ system does not exhibit any preferential orientation. For this reason the intensities and the line broadening in experimental 2D pattern display slight changes at different azimuthal angles, which is not the case in 2D calculated pattern (inset of Figure 4).

The microstructural analysis exhibits a roughly cubic crystallite shape for the $\mathrm{ZnS}$ phase with an average round dimension of $5 \mathrm{~nm}$. On the other hand, $\mathrm{ZnO}$ exhibits pseudodisc-shaped crystallite with the following dimension: $3.7 \mathrm{~nm}$ along the [011] and $3.1 \mathrm{~nm}$ along the [001] directions. These data were compared with the morphology and dimension of the coherent scattering domains as obtained from the DF TEM image, Image $\mathbf{J}$ processed, in Figure 5. The analysis of DF image, consisting in counting more than 200 crystalline scattering domain, provides a mean value for the crystallite size of $4 \mathrm{~nm}$ with associated standard deviations for the asymmetric statistical distribution of: $\sigma_{-}=1$ and $\sigma_{+}=3 \mathrm{~nm}$ (Figure 5). These values are of course in good agreement with the results obtained from the Rietveld method, with the strong limitation not to provide any information on the actual size and shape for the crystallites of each one of the identified

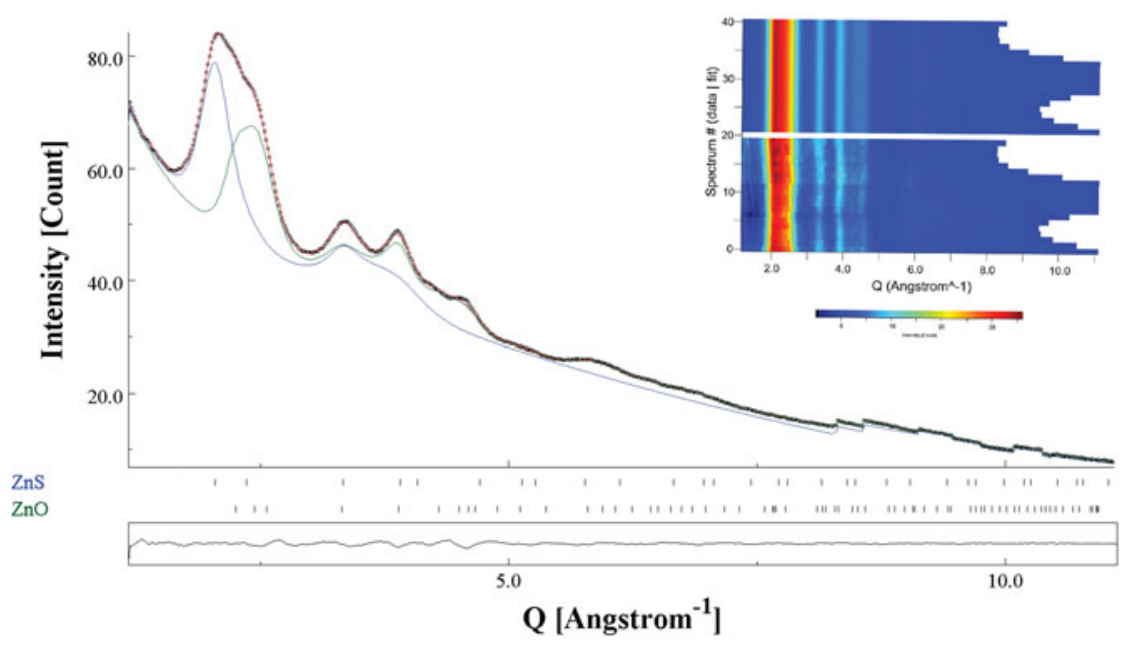

Figure 4. (Color online) Rietveld method applied to electron diffraction pattern of $\mathrm{ZnS} / \mathrm{ZnO}$ nanopowders. Dot line: Experimental intensity profile; red line: calculated profile; green line: $\mathrm{ZnO}$ intensity contribution (54.5\%); blue line: $\mathrm{ZnS}$ intensity contribution $(45.4 \%)$. In the inset the twodimensional multiplot of the calculated (upper part) and experimental (bottom part) profile are displayed. 

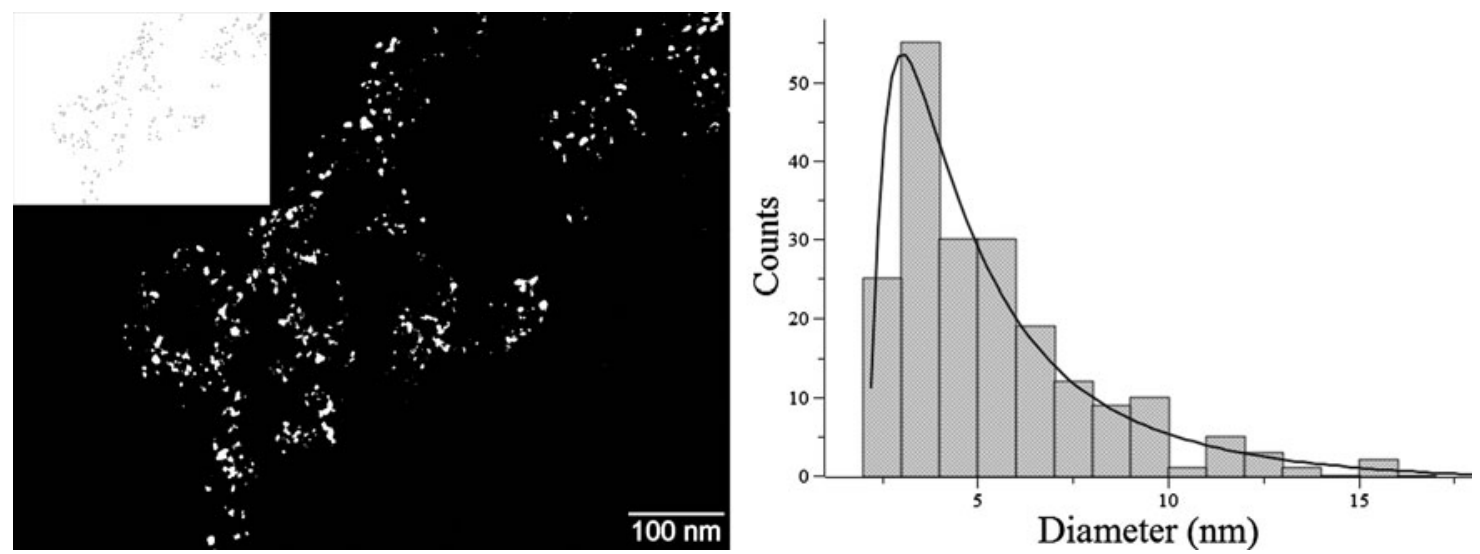

Figure 5. Dark Field Image Analysis on $\mathrm{ZnS} / \mathrm{ZnO}$ nanopowders aggregates: threshold of Dark Field TEM micrograph of the $\mathrm{ZnS} / \mathrm{ZnO}$ nanopowder aggregates (left); each ellipse in the image represent the analyzed particle (top left corner); histogram of size distribution of crystallites (right).

phases. The microstructural analysis was performed also using the XRD data (Figure 1). Crystallite size and shape for ZnS, the only detected phase, are in excellent agreement with the results obtained with the electron diffraction data (see Table I). One limitation of the diffraction analysis is that the shape information obtained can be different from the real one, as the refined shape is symmetrized by the Laue point group of the crystal structure as we cannot distinguish between equivalent crystallographic directions.

The Rietveld analysis of electron diffraction pattern is able to provide information on the content of crystalline phases inside the investigated volume. The $\mathrm{ZnS} / \mathrm{ZnO}$ system in the analyzed region shows that the volume fraction of the oxide $(55 \%)$ and the sulfide (45\%) are almost comparable (see Table I). Of course, these values do not correspond to the bulk phase content but to the local composition of the electron transparent regions displayed in the BF image in Figure 1. Phase quantification is highly dependent on the state of aggregation and actual local thickness of the agglomerates. From the analysis of different areas we confirm that the $\mathrm{ZnO}$ volume fractions are comparatively lower than $\mathrm{ZnS}$ in case of larger aggregates with higher thickness. An example of this situation is displayed in Figure 6 and relevant Table II, where the phase composition ( $\mathrm{ZnS}$ 69, $\mathrm{ZnO} 31 \%$ ) is significantly different from the previously analyzed zone. This experimental evidence suggests that, as expected, $\mathrm{ZnO}$ is prevalently present on the surface of the investigated areas in agreement with the proposed hypothesis of the formation of an ultrathin layer of oxide on the $\mathrm{ZnS}$ aggregates. Finally, as the results obtained through the electron diffraction have shown that the phase quantitative analysis data are highly dependent on the selection of the electron transparent region and, consequently, to the interaction volume.

\section{FINAL COMMENTS AND FURTHER DEVELOPMENTS}

Crystalline domain sizes and relevant shapes for both $\mathrm{ZnS}$ and $\mathrm{ZnO}$ phases were successfully evaluated using the Rietveld method applied to electron diffraction ring patterns. The results obtained from the microstructural characterization of nanostructured $\mathrm{ZnS} / \mathrm{ZnO}$ two-phase sample demonstrate the capabilities of this technique that may represent a fully quantitative method for the routine characterization of crystalline nanomaterials and of their outer layers. The proposed approach combines BF/DF-TEM and SAED analyses to improve the determination of size and shape of nanocrystalline domain ensembles. By applying the Rietveld method to the electron diffraction pattern a proper microstructural characterization is achieved even in case of agglomerated aggregates and multiphase samples. These samples, owing to the strong
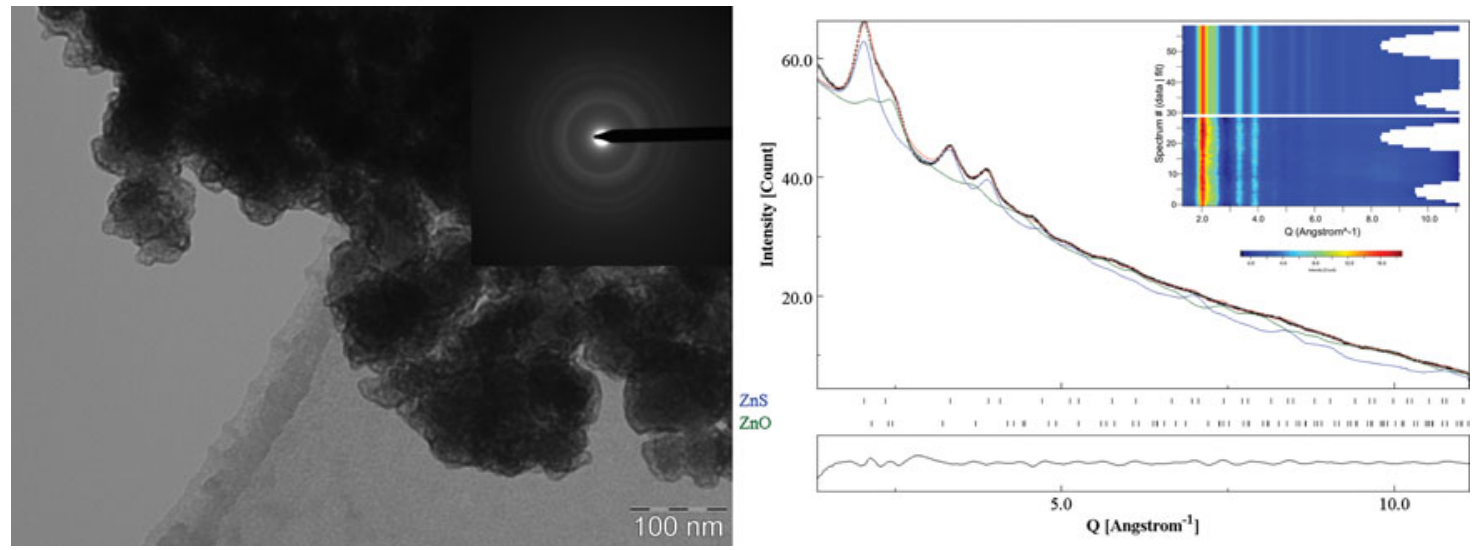

Figure 6. (Color online) TEM measurements on a larger area of $\mathrm{ZnS} / \mathrm{ZnO}$ system and higher average thickness as compared with the clusters in Figure 1: $\mathrm{BF}$ micrograph (left); SAED ring pattern (inset); Rietveld method applied to electron diffraction pattern (right). Dot line, experimental intensity profile; red line, calculated profile; green line, $\mathrm{ZnO}$ intensity contribution (39.2\%); blue line, $\mathrm{ZnS}$ intensity contribution (60.8\%). 
TABLE II. Results of electron diffraction pattern Rietveld analysis on thicker area (Figure 6). The numbers in parentheses denotes the estimated standard deviation in the last digit.

Reliability factor: $R_{\mathrm{w}}=2.60 \%$
Phase identification

$\mathrm{ZnS}$ sphalerite

ZnO Wurtzite

$60.8 \pm 0.8$

$39.2 \pm 0.5$ overlap of the broad diffraction components are not always easy to analyze using conventional TEM diffraction contrast. This work also shows the possibility of attaining phase identification and quantification at a nanoscale level. These features are not always achievable by common diffraction methods that investigate much larger volumes of material. Other advantages that can be envisaged for electron diffraction tools are: (1) comparatively fast data collection times: few seconds are needed for the SAED pattern complete acquisition; (2) very limited amount of sample necessary, commonly a few micrograms; (3) possibility of combining the electron diffraction data not only with images but also with chemical compositions, obtained by EDXS and/or EELS (electron energy loss spectroscopy) techniques, that can be all integrated in a combined data analysis approach.

\section{SUPPLEMENTARY MATERIAL}

The supplementary material for this article can be found at https://doi.org/10.1017/S0885715617000343

Baer, D. R., Gaspar, D. J., Nachimuthu, P., Techane, S. D., and Castner, D. G. (2010). "Application of surface chemical analysis tools for characterization of nanoparticles," Anal. Bioanal. Chem. 396, 983-1002.

Bell, N. C., Minelli, C., Tompkins, J., Stevens, M. M., and Shard, A. G. (2012). "Emerging techniques for submicrometer particle sizing applied to Stober silica," Langmuir 28, 10860-10872.

Blackman, M. (1939). "On the intensities of electron diffraction rings," Proc. R. Soc. Lond. A 173, 68-82.

Boullay, P., Lutterotti, L., Chateigner, D., and Sicard, L. (2014). "Fast microstructure and phase analyses of nanopowders using combined analysis of transmission electron microscopy scattering patterns," Acta Crystallogr. Sect. A, Found. Adv. 70, 448-456.

Dieckmann, Y., Cölfen, H., Hofmann, H., Petri-Fink, A., Hofmann, H., Cölfen, H., Dieckmann, Y., Cölfen, H., Hofmann, H., and Petri-Fink, A. (2009). "Particle size distribution measurements of manganese-doped ZnS nanoparticles," Anal. Chem. 81, 3889-3895.

Dolcet, P., Maurizio, C., Casarin, M., Pandolfo, L., Gialanella, S., Badocco, D., Pastore, P., Speghini, A., and Gross, S. (2015). "An effective twoemulsion approach to the synthesis of doped $\mathrm{ZnS}$ crystalline nanostructures,” Eur. J. Inorg. Chem. 4, 706-714.

Gemmi, M., Voltolini, M., Ferretti, A. M., and Ponti, A. (2011). "Quantitative texture analysis from powder-like electron diffraction data," J. Appl. Crystallogr. 44, 454-461.

Giorgetti, E., Marsili, P., Cicchi, S., Lascialfari, L., Albiani, M., Severi, M., Caporali, S., Muniz-Miranda, M., Pistone, A., and Giammanco, F. (2015). "Preparation of small size palladium nanoparticles by picosecond laser ablation and control of metal concentration in the colloid," J. Colloid Interface Sci. 442, 89-96.

Gražulis, S., Chateigner, D., Downs, R. T., Yokochi, A. F. T., Quirós, M., Lutterotti, L., Manakova, E., Butkus, J., Moeck, P., and Le Bail, A.
(2009). "Crystallography open database-an open-access collection of crystal structures", J. Appl. Crystallogr. 42(4), 726-729.

Hassellöv, M., Readman, J. W., Ranville, J. F., and Tiede, K. (2008). "Nanoparticle analysis and characterization methodologies in environmental risk assessment of engineered nanoparticles," Ecotoxicology 17, 344-361.

Ischia, G., Wenk, H.-R., Lutterotti, L., and Berberich, F. (2005). "Quantitative Rietveld texture analysis of zirconium from single synchrotron diffraction images," J. Appl. Crystallogr. 38, 377-380.

Jain, P. K., Huang, X., El-Sayed, I. H., and El-Sayed, M. A. (2008). "Noble metals on the nanoscale: optical and photothermal properties and some applications in imaging, sensing, biology, and medicine," Acc. Chem. Res. 41, 1578-1586.

Kim, J. G., Seo, J. W., Cheon, J., and Kim, Y. J. (2009). "Rietveld analysis of nano-crystalline $\mathrm{MnFe}_{2} \mathrm{O}_{4}$ with electron powder diffraction," Bull. Korean Chem. Soc. 30, 183-187.

Klaine, S. J., Alvarez, P. J. J., Batley, G. E., Fernandes, T. F., Handy, R. D., Lyon, D. Y., Mahendra, S., McLaughlin, M. J., and Lead, J. R. (2008). "Nanomaterials in the environment: behavior, fate, bioavailability, and effects.," Environ. Toxicol. Chem. 27, 1825-1851.

Lábár, J. L. (2008). "Electron diffraction based analysis of phase fractions and texture in nanocrystalline thin films. I. Principles," Microsc. Microanal. 14, 287-295.

Lábár, J. L. (2009). "Electron diffraction based analysis of phase fractions and texture in nanocrystalline thin films, part II: implementation," Microsc. Microanal. 15, 20-29.

Lábár, J. L., Adamik, M., Barna, B. P., Czigány, Z., Fogarassy, Z., Horváth, Z. E., Geszti, O., Misják, F., Morgiel, J., Radnóczi, G., Sáfrán, G., Székely, L., and Szüts, T. (2012). "Electron diffraction based analysis of phase fractions and texture in nanocrystalline thin films. III. Application examples," Microsc. Microanal. 18, 406-420.

Lascialfari, L., Marsili, P., Caporali, S., Muniz-Miranda, M., Margheri, G., Serafini, A., Brandi, A., Giorgetti, E., and Cicchi, S. (2014). "Carbon nanotubes/laser ablation gold nanoparticles composites," Thin Solid Films 569, 93-99.

Linkov, P., Artemyev, M., Efimov, A. E., and Nabiev, I. (2013). "Comparative advantages and limitations of the basic metrology methods applied to the characterization of nanomaterials," Nanoscale 5, 8781-8798.

Linsinger, T., Roebben, G., Gilliland, D., Calzolai, L., Rossi, F., Gibson, N., and Klein, C. (2012). Requirements on Measurements for the Implementation of the European Commission Definition of the Term 'Nanomaterial (JRC Reference Report, EUR 25404 EN).

Lutterotti, L. (2010). "Total pattern fitting for the combined size-strainstress-texture determination in thin film diffraction", Nucl. Instrum. Methods Phys. Res.: B, Beam Interact. Mater. Atoms 268(3), 334-340.

Lutterotti, L., Bortolotti, M., Ischia, G., Lonardelli, I., and Wenk, H. R. (2007). "Rietveld texture analysis from diffraction images," $\mathrm{Z}$. Kristallogr. Suppl. 26, 125-130.

Lutterotti, L., Vasin, R., and Wenk, H. R. (2014). "Rietveld texture analysis from synchrotron diffraction images. I. Calibration and basic analysis," Powder Diffraction 29(1), 76-84.

Peng, L. M., Ren, G., Dudarev, S. L., and Whelan, M. J. (1996). "Robust parameterization of elastic and absorptive electron atomic scattering factors," Acta Crystallogr. Sect. A Found. Crystallogr. 52, 257-276.

Popa, N. C. (1998). "The $(h k l)$ dependence of diffraction-line broadening caused by strain and size for all Laue groups in Rietveld refinement," J. Appl. Crystallogr. 31, 176-180.

Rietveld, H. M. (1969). "A profile refinement method for nuclear and magnetic structures," J. Appl. Crystallogr. 2, 65-71.

Schneider, C. A., Rasband, W. S., and Eliceiri, K. W. (2012). "NIH Image to ImageJ: 25 years of image analysis," Nat. Methods 9, 671-675.

Silvestre, C., Duraccio, D., and Cimmino, S. (2011). "Food packaging based on polymer nanomaterials," Prog. Polym. Sci. 36, 1766-1782.

Simon, P. and Gogotsi, Y. (2008). "Materials for electrochemical capacitors," Nat. Mater. 7, 845-854.

Yetisen, A. K., Qu, H., Manbachi, A., Butt, H., Dokmeci, M. R., Hinestroza, J. P., Skorobogatiy, M., Khademhosseini, A., and Yun, S. H. (2016). "Nanotechnology in textiles," ACS Nano 10, 3042-3068. 